\title{
L'appareil disciplinaire au collège en France : un opérateur hiérarchique de genre
}

The disciplinary apparatus in secondary schools in France: A hierarchical operator of gender

El aparato disciplinar en el colegio en Francia: un operador jerárquico de género

Sylvie Ayral

\section{(2) OpenEdition}

\section{Journals}

Édition électronique

URL : https://journals.openedition.org/ries/8730

DOI : 10.4000/ries.8730

ISSN : 2261-4265

Éditeur

France Education international

Édition imprimée

Date de publication : 1 septembre 2019

Pagination : 71-80

ISBN : 978-2-85420-624-1

ISSN : $1254-4590$

Référence électronique

Sylvie Ayral, «L'appareil disciplinaire au collège en France : un opérateur hiérarchique de genre », Revue internationale d'éducation de Sèvres [En ligne], 81 | septembre 2019, mis en ligne le 01 septembre 2021. consulté le 14 octobre 2021. URL : http://journals.openedition.org/ries/8730 ; DOI : https://doi.org/ $10.4000 /$ ries. 8730 


\title{
L’appareil disciplinaire au collège en France : un opérateur hiérarchique de genre
}

\author{
Sylvie Ayral \\ Professeure agrégée
}

En France, promouvoir l'égalité des filles et des garçons constitue une obligation légale et une mission fondamentale pour l'éducation nationale ${ }^{1}$. Les textes officiels préconisent une culture de l'égalité : le référentiel des compétences professionnelles des métiers du professorat et de l'éducation ${ }^{2}$ demande explicitement aux personnels de «se mobiliser et mobiliser les élèves contre les stéréotypes et les discriminations de tout ordre, promouvoir l'égalité entre les filles et les garçons, les femmes et les hommes ». Le code de l'éducation rappelle que des actions doivent être menées pour cela dès l'école primaire et que l'égalité doit se décliner dans le quotidien de la classe. Les politiques académiques accompagnent les équipes éducatives en proposant désormais des actions de formation vers tous les acteurs de l'école et leurs sites internet affichent de très nombreuses ressources pédagogiques...

Et pourtant... Pour une institution qui se veut égalitaire, un constat embarrassant persiste : en 2009, nous avions montré dans le cadre d'une thèse de doctorat (Ayral, 2011) que $80 \%$ des élèves punis ${ }^{3}$ au collège étaient des garçons, tous motifs confondus. Et ceci dans toutes les catégories d'établissements, qu'ils soient privés ou publics, de centre-ville, de la banlieue ou du milieu rural. Les garçons représentaient encore $83 \%$ des élèves punis pour indiscipline ou insolence et $91,7 \%$ de ceux sanctionnés pour des actes relevant d'atteinte aux biens et aux personnes. Ces chiffres, très peu interrogés jusqu'alors, ont été confirmés depuis à maintes reprises. Les équipes éducatives les considèrent comme naturels (" une question de gènes ou d'hormones ", en contradiction avec toutes les études scientifiques contemporaines, qui démontrent le caractère majoritairement construit des identités de genre) ou déterminés par les milieux socioculturels.

Dix ans plus tard, rien n'a changé : dans le cadre des nombreuses conférences et formations que j'assure auprès des acteurs éducatifs, je demande aux établissements de bien vouloir me communiquer leurs statistiques sexuées en matière de sanctions et punitions : la surreprésentation masculine reste identique...

1. Convention-loi $\mathrm{n}^{\circ} 2013$ du 3 juillet 2013 d'orientation et de programmation pour la refondation de l'école de la République.

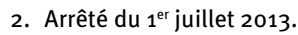

3. Les punitions scolaires sont des mesures d'ordre intérieur. Elles concernent les manquements mineurs aux obligations des élèves et les perturbations ponctuelles de la vie de la classe ou de l'établissement. Les sanctions scolaires concernent les atteintes aux personnes ou aux biens ainsi que des manquements graves ou répétés aux obligations des élèves. Elles sont inscrites au dossier administratif de l'élève et peuvent faire l'objet d'un recours devant les tribunaux administratifs. Je n'établirai pas de distinction entre punition et sanction scolaire dans cet article et emploierai indifféremment l'un ou l'autre terme. 
Comment l'expliquer ? Pourquoi et surtout comment perdure un tel décalage entre un discours officiel, qui réaffirme le principe de l'égalité des sexes, et des pratiques punitives qui en prennent le contre-pied, alors que la sanction se veut, depuis maintenant des années, " éducative » et que les effets négatifs des punitions données de manière excessive sont démontrés depuis longtemps ? L'hypothèse que le système punitif constitue en soi l'un des vecteurs de la construction et de la reproduction des inégalités sexuées à l'école ne peut être écarté. Étudier la sanction comme un opérateur ${ }^{4}$ agissant au cœur du système permet de mieux appréhender son rôle et de comprendre comment s'institue l'ordre de genre à l'école. La sanction, qui consacre le pouvoir qui punit dans une institution restée patriarcale ${ }^{5}$, opère matériellement la distinction et la hiérarchisation entre les sexes. Elle participe au maintien, de façon quasi invisible, de l'ordre de genre et produit les violences qui lui permettent de se perpétuer, rendant d'autant moins efficaces les mesures prises pour l'égalité entre filles et garçons.

\section{LA SANCTION, OPÉRATEUR HIÉRARCHIQUE}

La sanction disciplinaire pose la question de sa propre justification : elle s'accompagne de discours de légitimation qui justifient à l'avance la coercition et la répression punitive exercée par la classe adulte sur la classe la plus jeune, tout en témoignant de l'évolution des concepts d'éducation, d'enfance et d'adolescence.

En France, Claude Allègre, ministre de l'éducation nationale, de la recherche et de la technologie en 2000, pose le principe d'une lecture juridique des faits d'indiscipline. La sanction devra être éducative et respecter les quatre principes du droit pénal : proportionnalité, légalité, individualisation, contradictoire. La réglementation de juillet 2000 affirme ainsi la valeur formatrice et pédagogique de la sanction, qui devra désormais poursuivre une double finalité : responsabiliser l'élève et lui rappeler le sens et l'utilité de la loi. En 2008, le ministre Xavier Darcos établit le code des règles de vie scolaire et instaure le conseil de discipline. Il invite à rechercher systématiquement la responsabilité civile et encourage le recours à l'exclusion. Plus récemment le $\mathrm{BO}^{6} \mathrm{n}^{\circ} 35$ du 27 septembre 2018, rédigé sous le ministère de Jean-Michel Blanquer, rappelle l'importance des mesures de prévention et d'accompagnement et reprend la " mesure de responsabilisation » qui consiste pour l'élève à participer, en dehors des heures d'enseignement, à des activités de solidarité, culturelles ou de formation à des fins éducatives.

Dans ses bases juridiques, la sanction est liée à l'existence d'un règlement intérieur (RI) qui doit lui réserver un chapitre. Le RI est approuvé et voté par le conseil d'administration de chaque établissement Les discours de légitimation y apparaissent sous forme de préambules ( Sa raison d'être est de favoriser d'une part, la réussite scolaire des élèves et d'autre part, leur insertion professionnelle »),

4. Le terme « opérateur » ou « actant » désigne « toute entité définissable et distinguable qui participe à la dynamique et à l'organisation d'une action individuelle ou/et collective, qui est active dans un processus social, qui opère des actes (Latour, cité par Lévy et Lussault, 2003).

5. Dans sa hiérarchie, ses modes de fonctionnement, son organisation administrative, etc. (Ayral, 2011).

6. Bulletin officiel de l'Education nationale 
qui renvoient à des lois, des décrets, des délibérations prises au sein d'instances supérieures ( Vu les traités internationaux ratifiés par la France en matière d'éducation et de protection des individus / Vu la constitution de la $\mathrm{V}^{\circ}$ République / Vu la loi d'orientation 89-406 du 10 juillet $1989 »)$. C'est un début impressionnant pour des élèves et des familles qui, de toute façon, seront dans l'obligation d'apposer leur signature à la fin... Le champ lexical est celui de la légitimation : « Tout manquement caractérisé au règlement intérieur justifie la mise en œuvre d'une procédure disciplinaire ou de poursuites appropriées »; «La commission Vie scolaire est adaptée et pertinente pour arbitrer les cas les plus graves ». Les RI se déclinent sur le mode de l'obligation et de la coercition (le verbe « devoir », le substantif « obligation », les adjectifs « obligatoire » ou « impératif », les adverbes « obligatoirement » et « impérativement » jalonnent le texte) ou de l'interdiction (" il est interdit de », " il est strictement interdit de », " il est formellement interdit de »), même si elle est parfois euphémisée ( « les élèves ne sont pas autorisés à », " aucun élève n’a le droit de », etc.).

L'appareil d'écriture inscrit, en creux, la définition de l'élève idéal, la contrainte d'une conformité à réaliser (Foucault, 1975). Enfreindre le règlement, ne pas se conformer aux normes et donc aux attentes qu'il énonce, c'est prendre le risque d'être sanctionné, stigmatisé, catalogué comme déviant (Becker, 1963). Or, d'un collège à l'autre, l'appareil punitif fonctionne de façon totalement différente, de la qualification divergente des infractions au choix arbitraire de la sanction. Dans tel établissement, on décide tout à coup d'accentuer la répression, dans d'autres, on constate un relâchement disciplinaire à certaines périodes de l'année ou encore un respect approximatif des principes du droit pénal, par exemple pour les violences sexuelles (Mercader et al., 2016). Une fois institué, l’appareil disciplinaire se déploie parfois fort loin des situations pédagogiques, fonctionnant de manière quasi autonome : c'est un opérateur qui met en scène la hiérarchie des pouvoirs.

Le vocabulaire utilisé dans le chapitre "sanctions disciplinaires » emprunte souvent au champ lexical juridico-judiciaire comme en témoigne cette lettre d'une conseillère principale d'éducation (CPE) d'un collège à la famille d'un élève :

En acceptant un stylo qui à l'origine a été volé, R. s'est mis dans une situation contraire à la loi : Art. 311-3 du Code pénal. Il a été convenu que la sanction restera à l'interne, mais que si une autre histoire du même genre intervenait, cela sortirait de l'établissement (par dépôt de plainte). En conséquence, un travail de réflexion devra être remis à la vie scolaire pour le 30 avril.

Rappel de la loi, citation de l'article du code pénal correspondant, intimidation, menaces... jusqu'à la « peine » qui « tombe » : le pouvoir qui punit s'exprime dans tout son éclat. Les rapports d'incident des enseignants et les procès-verbaux de conseils de discipline en collège ressemblent à s'y méprendre à ce que l'on pourrait trouver au bas d'une signification de jugement pénal :

«La mesure d'exclusion est assortie du sursis avec une période probatoire de 3 mois ».

«L'élève L. J. s'est rendu coupable de menaces avec extorsion de résultats d'interrogation écrite».

«Extorsion de devoir envers un camarade de sa classe ».

«S'est rendu coupable de faux témoignage».

" Comportement perturbateur et provocateur avec attitude récidiviste».

«Vol de trousse en groupe », etc. 
Les professeurs et assistants d'éducation qui rencontrent des difficultés disciplinaires graves avec un élève règlent rarement leur problème eux-mêmes : le plus souvent, ils le font accompagner au bureau du/de la CPE voire du/de la principal(e) et sollicitent une sanction. Cette pratique rend lisibles les rapports hiérarchiques internes en consacrant premièrement l'autorité du/de la chef(fe) d'établissement sur la communauté des adultes, deuxièmement le pouvoir punitif accru du/de la CPE par rapport à celui des enseignants ou assistants d'éducation. En voici une illustration : c'est un mail envoyé en février 2019 par une professeure principale à l'ensemble de l'équipe pédagogique ${ }^{7}$ (je faisais partie des destinataires) :

« Nous avons reçu Thomas en entretien ce matin avec Marie [une autre professeure] : il a nié la tricherie en $\mathrm{SVT}^{8}$ au départ puis nous le lui avons fait avouer par écrit [...]. Le chef [d'établissement] est prévenu. Il va être sanctionné. Nous allons avoir un rendez-vous avec le chef. Marie et moi voulons 2 jours d'exclusion ferme : à négocier...»

L'attribution d'une sanction est donc un dispositif autonome ritualisé, renforcé par l'appareil d'écriture qui l'accompagne. Ce dispositif désigne et stigmatise le contrevenant en même temps qu'il met en scène la hiérarchie des pouvoirs. Nous allons voir maintenant comment la sanction scolaire joue un rôle stratégique dans la construction et le renforcement de l'identité masculine des garçons et des adultes hommes afin de se différencier hiérarchiquement des filles pour les premiers, des collègues femmes pour les seconds, ainsi que des garçons ou des hommes les plus faibles.

\section{LA SANCTION, OPÉRATEUR HIÉRARCHIQUE DE GENRE}

\section{Se démarquer hiérarchiquement du féminin}

L'enquête qualitative, dans une perspective compréhensive, permet d'analyser le sens que les acteurs donnent à leurs pratiques et fait émerger les systèmes de valeur et les repères normatifs à partir desquels ils s'orientent et se déterminent. Lorsqu'on donne la parole à des garçons de collège (Ayral, 2011) et qu'on leur demande comment ils expliquent que $80 \%$ des élèves punis au collège soient des pairs, ils fanfaronnent et cherchent à se démarquer des filles :

«Les filles, quand elles sont collées, elles pleurent... Nous on n'a pas peur de faire des bêtises" (élève de $4^{\mathrm{e}}$ ).

Elisabeth Badinter (1992) explique que « l'homophobie (en tant que haine des qualités féminines chez les hommes), à l'instar de la misogynie, joue un rôle important dans le sentiment d'identité masculine. Être un homme signifie ne pas être féminin, ne pas être homosexuel ».

7. Les prénoms ont été changés.

8. Sciences de la vie et de la terre. 
La transgression du règlement intérieur est la plupart du temps un acte volontaire comme nous l'explique cet élève de $5^{\mathrm{e}}$, régulièrement sanctionné :

«Moi, je suis exclu bientôt trois jours, mais justement, je suis content de partir parce que [...] c'est vrai que d'avoir des punitions ou d'être renvoyé, c'est... pour moi c'est quelque chose aussi de bien, parce que bon, c'est... ça, ça donne des adrénalines et ça donne des pulsations au cœur et on sait pas ce qui nous attend, donc, ça nous fait quelque chose vraiment de fort quoi. [...]. C'est ce que je recherche».

Si le premier avantage de la punition est clairement le plaisir éprouvé pendant le processus d'infraction, le second semble être le rôle positif qu'elle joue dans la conquête des filles, instruments de luttes symboliques pour démontrer et comparer sa virilité (Bourdieu, 1998). Le but est de se faire remarquer à tout prix par le public féminin et de prouver au passage son appartenance au groupe des "vrais hommes ", en montrant son " potentiel supérieur de courage », c'est-à-dire, métaphoriquement, ses attributs sexuels. Ainsi une élève interviewée déclare à propos d'un garçon qui avait osé défier une assistante d'éducation :

«Lui, il a des couilles, enfin... en quelque sorte, [...] il ose.» (Élève de $3^{\mathrm{e}}$ ).

La sanction fonctionne alors comme un rite sexué et sexuant et sa quête relève de la parade amoureuse (Goffman, 1977). Il s'agit bien de conduites sociales, non de problèmes, et encore moins de troubles du comportement.

La sanction est une épreuve, au sens où elle implique une certaine souffrance (peur qui participe de l'effet de passage, admonestation de l'enseignant, convocation dans le bureau des CPE ou du/ de la principal(e), sermon des parents). Mais le jeu en vaut la chandelle. Pour les garçons punis et aux yeux de leurs pairs, la sanction qui tombe est une consécration, une médaille de virilité. Ceci est d'autant plus valable lorsqu'ils défient l'autorité, qu'ils insultent les assistantes d'éducation ou les professeures, en les humiliant par des remarques ouvertement sexistes ${ }^{9}$. Le but, alors, est d'inverser le rapport hiérarchique, de rabaisser l'enseignante ou l'assistante d'éducation à sa condition de femme, donc d'objet sexuel. Ces pratiques sont courantes et alimentent de nombreuses demandes de sanctions. Le récit qui suit est celui d'une professeure d'éducation physique et sportive (EPS) :

"Une fois dans un vestiaire, il y avait eu du bruit, $\mathrm{j}$ 'avais dit à travers la porte « dépêchez-vous de sortir, taisez-vous, dépêchez-vous » " y a un élève qui a dit " mais si vous voulez, vous pouvez entrer me la sucer », [...] donc bon là, on a fini par savoir qui était l'élève, il a été exclu 15 jours ».

En osant tenir de tels propos, ce garçon prouve à la communauté des élèves qu'il est capable de réaliser ce qu'aucun d'eux n'aurait osé faire. Il donne ainsi la preuve qu'il domine symboliquement non seulement les femmes (par la sexualité, même fantasmée) mais également les autres garçons, ceux qui ne sont pratiquement jamais punis et qu'il considère volontiers comme des « bouffons » ou des «tapettes ».

9. Le sexisme est une idéologie qui repose sur l'idée que les femmes sont inférieures aux hommes. Ses manifestations sont très diverses, clichés, blagues, remarques jusqu'aux formes les plus graves (discriminations, mépris, hostilité, violences, meurtre). Le sexisme nie le droit à l'égalité des êtres humains. Des caractéristiques et des rôles spécifiques dans la société sont attribués à l'un et l'autre sexe, de manière arbitraire, répétée et surtout asymétrique, où tout ce qui est assimilé au féminin est dénigré et rabaissé. 
Dans tous les collèges enquêtés pour mon travail de recherche et ainsi qu'en témoigne le rapport 2018 de l'Observatoire européen de la violence à l'école (Debarbieux et al., 2018), les relations entre garçons sont marquées par les humiliations sexuelles et par l'homophobie. Les rapports écrits par les adultes et consignés dans les registres des sanctions de différents établissements témoignent de cette oppression quotidienne :

«En début de contrôle, J. s'aperçoit que son blouson a été sali (peut-être par inadvertance) par A., qui était à la table derrière lui. Il se retourne alors en vociférant des insultes : "Espèce de gros pédé, je vais te niquer ; tu me casses les couilles" " (Professeure d'éducation musicale).

«L. $\left(5^{\mathrm{e}}\right)$ est exclu de cours, il doit être assis à la table devant la vie scolaire mais il se lève sans cesse et perturbe la permanence. Il se bat avec M. et lui dit: "je te plante, va te faire foutre, je t'encule". » (Assistante d'éducation).

On est bien, à chaque fois, dans l'imaginaire d'une sexualité de domination car si, sous sa forme active, l'homosexualité peut être considérée par l'homme comme un moyen d'affirmer sa puissance, sous sa forme passive elle est au contraire un symbole du féminin et donc de la déchéance (Reynaud, 1981). La sanction rend visible la hiérarchie de genre à l'intérieur même de la catégorie "garçons ». Ceux qui sont punis, les dominants violents, sexistes et homophobes présentent des comportements conformes aux normes de la virilité, c'est-à-dire au sexe prescrit. Les autres sont déclassés dans la catégorie des sous-hommes, c'est-à-dire des faibles, des pédés et deviennent les cibles potentielles des premiers.

\section{De l'autorité masculine à la supériorité du masculin}

L'autorité éducative et pédagogique peut être lue, elle aussi, à la lumière des rapports sociaux de sexe. La sanction opère de la même façon chez les adultes, révélant des rapports à l'autorité différenciés entre femmes et hommes mais également au sein de chaque catégorie. Les femmes estiment avoir plus de difficultés à se faire respecter et à maintenir la discipline que leurs collègues masculins, du fait de leur petite taille, de leur inaptitude au combat physique et du peu de crédit porté à l'autorité féminine. Une professeure de lettres explique :

«- Je pense que les gamins quand ils ont en face d'eux un mec grand et pêchu parce qu'il est encore jeune et ben ils ont tendance à moins la ramener.

- Question : Ils ont peur de quoi, concrètement?

- Et bien le rapport de force, de force... physique, évidemment ! En conseil de classe les profs hommes ont beaucoup moins souvent des problèmes d'autorité, se plaignent beaucoup moins souvent d'agitation dans la classe ».

D’où vient cette autorité masculine ? Les hommes interrogés disent ne pas hésiter, au besoin, à entretenir le fantasme d'une confrontation physique pour impressionner les garçons provocateurs et asseoir leur autorité. Un enseignant raconte :

« J'ai proposé à l'élève de le retrouver sur le trottoir, lui avec ses copains et moi avec les miens. Il n'y a pas eu de suite. » (Professeur d'histoire-géographie). 


\begin{abstract}
Morceau choisi :
« Cela dit j'ai déjà utilisé la dissuasion physique pas en le transformant en contact mais en le transformant en jeu. J'avais une fois dans une classe un grand. Un grand, vraiment, qui était, qui avait fait [...] sa poussée de croissance et qui était imposant et qui martyrisait les autres garçons. [...] Je lui ai dit : "écoute, ça suffit, si tu veux avoir un adversaire à ta taille je suis là ! Et on va faire un jeu." Et on l'a fait devant la classe. C'était un truc où je savais que je gagnerais. C'était un jeu très simple : on se prend les mains comme ça, pour faire plier l'autre avec les poignets. [...] Et je l'ai fait plier, je l'ai fait mettre à genoux. Et ça a été euh... un peu dans l'amusement, un peu genre arènes de théâtre, arènes de... euh... romaines et donc il a perdu. [...] Il avait perdu son statut d'homme quoi [...] C'était pour l'émousser vis-à-vis des autres garçons » (professeur de technologie).»
\end{abstract}

L'enseignant tente de minimiser l'anecdote en la présentant comme un jeu mais ce n'en est pas moins une démonstration de virilité éclatante. Il présente l'élève comme étant " un grand, vraiment », " qui martyrisait les autres garçons » (donc puissant, violent et potentiellement dangereux) et se présente comme " un adversaire à sa taille ». Il est, de toutes façons, certain d'être d'une force supérieure («J'ai de la force dans les poignets donc je savais que je gagnerais »). Le spectacle de la puissance virile peut alors commencer ( « on l'a fait devant la classe »; " un peu genre arènes de théâtre »). En humiliant ce jeune garçon ( «Il avait perdu son statut d'homme »; " c'était pour l'émousser vis-à-vis des autres garçons »), l'enseignant est lui-même reconnu et conforté dans sa virilité : plus besoin de sanctionner...

Cependant, tous les enseignants masculins ne se lancent pas dans ce genre de démonstration pour assoir leur autorité, loin s'en faut : un documentaliste évoque, lors d'un entretien, un phénomène fréquent, celui d'enseignants hommes en difficulté face à leurs élèves : "Y en a certains qui s'en vantent pas. C’est dramatique ». Ces hommes évitent de sanctionner pour que cela ne se sache pas : un homme est supposé avoir une autorité naturelle, un potentiel de force physique qui devrait en principe suffire pour s'imposer après des élèves. Avouer ses difficultés pour un enseignant masculin est de l'ordre du tabou, de l'indicible : ce serait se couvrir de honte et être déclassé.

Le mythe d'une autorité naturelle chez les hommes est le fruit d'un travail incessant de reproduction auquel contribuent les hommes (en entretenant le fantasme d'une confrontation physique toujours possible lorsqu'ils sont dominants) mais aussi les femmes, qui ont incorporé cette domination par habitus (Bourdieu, 1998). La sanction confère finalement une importance chaque jour renouvelée au masculin : à l'autorité masculine, comme cela vient d'être évoqué mais aussi aux garçons punis, qu'ils soient désignés comme des modèles masculins négatifs par l'institution ou comme des modèles masculins positifs par la communauté des pairs.

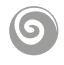

Dès lors que l'on appréhende l'appareil disciplinaire comme un opérateur hiérarchique, on ne peut plus se contenter de considérer l'asymétrie des sanctions distribuées aux garçons et aux filles comme un simple reflet du monde qui 
nous entoure mais comme étant, au moins autant, la production d'une institution patriarcale. Le concept d'opérateur permet de mettre en lumière le rôle à la fois dynamique et symbolique de la sanction dans les disparités sexuées que le système scolaire produit ou perpétue. L'appareil disciplinaire constitue à lui seul un système diviseur signifiant qui structure fortement l'organisation sociale du collège : il est un opérateur hiérarchique de genre.

Il est un opérateur hiérarchique parce que qu'il dicte les rapports entre les différents acteurs, fixant des limites à l'expérience sociale de chacun.

Il est un opérateur de genre parce qu'il trie les élèves et les enseignants en fonction de leur sexe et de la conformité de leurs comportements aux rôles sociaux attribués à chacun. Non seulement les garçons sont quatre fois plus punis, mais ils le sont pour des motifs sexués masculins (indiscipline, insolence, incivilités, dégradations, violence sur autrui). Il distingue et consacre les garçons quantitativement et qualitativement en même temps qu'il invisibilise les filles. En enregistrant la reproduction des standards de genre, les registres des établissements opèrent comme des boîtes noires qui font apparaître comme naturels les comportements sexués des adolescents et des adultes qui les encadrent.

Enfin, l'appareil disciplinaire fonctionne comme un opérateur hiérarchique de genre parce que, en contradiction totale avec les discours totalitaires officiels, il rend visible et met en scène le statut supérieur du masculin sur l'ensemble du « fait social total » qu'est le collège. Il constitue ainsi un vecteur majeur d'inégalité sexuée.

En France, l'école est un lieu de tensions importantes pour amener l'enfant à modifier son comportement. Les notions de punition et de sanction semblent inséparables de celle d'éducation. Il y a toujours eu, dans la culture disciplinaire scolaire, un vaste répertoire de pratiques et d'instruments punitifs, particulièrement dans l'enseignement primaire et au collège.

Au début du $\mathrm{xx}^{\mathrm{e}}$ siècle, les défenseurs d'une école libertaire ont inquiété plus qu'ils n'ont modifié réellement les pratiques pédagogiques et punitives. Plus tard, les événements de mai 1968 ont contribué à la remise en cause de l'enseignement et des pratiques punitives : une partie de la société s'est sentie en manque de repères, estimant que l'on était allé trop loin dans l'abolition de l'autorité. Les années 1990 ont marqué le retour de thèmes que l'on croyait tombés en désuétude : autorité, loi, sanction, en insistant sur leur dimension socialisante. Cela n'a pas empêché l'augmentation des faits d'indiscipline et de violence à l'école. Au $\mathrm{XxI}^{\mathrm{e}}$ siècle et à l'heure où l'on s'intéresse de plus en plus aux vertus d'un climat scolaire apaisé, à l'éducation bienveillante, à la réussite de tous les élèves et à l'égalité réelle entre les filles et les garçons, ne serait-il pas grand temps de mener une réflexion de fond sur nos pratiques punitives?

\section{RÉFÉRENCES BIBLIOGRAPHIQUES}

AYRAL S. (2011). La fabrique des garçons. Sanctions et genre au collège. Paris : PUF.

BADINTER E. (1992). XY, de l'identité masculine. Paris : Odile Jacob

BECKER H.-S. (1985 [1963]). Outsiders. Études de sociologie de la déviance. Paris : Métailié. BOURDIEU P. (1998). La domination masculine. Paris : Liber Seuil 
DEBARBIEUX E., ALESSANDRIN A., DAGORN J. et GAILLARD O. (2018). Les violences sexistes à l'école. Une oppression viriliste. Paris : Observatoire européen de la violence à l'école. [En ligne] https://www.nogynophobie.org/media/rapport-violences-sexiste-ecole-2018.pdf FOUCAULT M. (1975). Surveiller et punir. Paris : Gallimard.

GOFFMAN E. (2002 [1977]), [tr. fr]. L'arrangement des sexes. Paris : La Dispute.

LUSSAULT M., (2013., " Opérateur et Actant », in J. Lévy et M. Lussault, Dictionnaire de la géographie, Paris, Belin, p. 736-738.

MERCADER P., LECHENET A., DURIF-VAREMBONT J.-P. et GARCIA M.-C. (dir.) (2016). Mixité et violence ordinaire au collège et au lycée. Paris : Erès

REYNAUD E. (1981). La sainte virilité. Paris : Syros. 
